\title{
SYMPTOMATIC ARTERIAL HYPERTENSION IN CHILDREN - CLINICAL OBSERVATION
}

\author{
Marinov L., D. Bliznakova, P. Shivachev \\ Second specialized pediatric clinic of cardiology, rheumatology, nephrology, \\ gastroenterology and intensive care, University hospital "St. Marina" - Varna
}

Reviewed by: Assoc. Prof. Iv. Galabov

\begin{abstract}
The Symptomatic Arterial Hypertension (SAH) is found rarely compared to the essential. Its incidence is approximately $15-20 \%$ of the whole frequency of the hypertension conditions. From age aspect, as lower is the age on which the high arterial blood pressure is observed, the probability that it is SAH is higher. SAH is a symptom of another already existing disease, which causes the rising of the blood pressure. Most often these are inherited or acquired diseases of the urinary system, of the cardiovascular system, of the endocrine and of the nerve system. The aim of this study is to specify the frequency and etiological belonging of SAH in hospitalized children. From the 134 children with high blood pressure, hospitalized for eight years, in 14,18\% we found that this is a SAH. The highest is the number of children with renal parenchimal disease - 14, with renal polycystosis $\mathbf{- 3}$, with stenosis of the renal arteries $\mathbf{- 2}$ and one child with suprarenal tumor. Two of the children with parenchimal disease were in advanced stages, with developed renal insufficiency, and in one of them we had to start haemodialysis. The children with SAH had high levels of blood pressure; some of them were hospitalized after hypertonic crisis, with signs of hypertonic encephalopathy, which were the initial signs of the high blood pressure. SAH, even rare during childhood, is a serious disease, leading to life threatening complications, requiring fast diagnosis and adequate treatment.
\end{abstract}

Key words: symptomatic arterial hypertension, hypertonic crisis, children

The symptomatic arterial hypertensions are found significantly rare, compared to the primary arterial hypertension. Their incidence is $15-20 \%$ from the whole frequency of the hypertension conditions. From age aspect, as smaller is the child with high arterial blood pressure, the probability that it is Symptomatic Arterial Hypertension (SAH) is higher. $\mathrm{SAH}$ is a symptom of already existing disease. Most often these are diseases of the urinary system, inherited or acquired, diseases of the cardiovascular, endocrine, nerve system, iatrogenous hypertensions, etc. $(1,3,4)$.

After conduction of treatment according to the "rules of the art" and there is no effect or insufficient answer of the level of the blood pressure, usually is suspected the presence of $\mathrm{SAH}$. Then there is a necessity to find out the etiology of the hypertension.

Recently in the clinical practice we had such cases, in which we thought that these were children with essential hypertension, but because of inadequate answer to the conducted treatment, we had to widen the diagnostic methods and in some cases we found other primary diseases, in which the high blood pressure was a main symptom. The aim of this study is to specify the frequency and etiological belonging of SAH in hospitalized children.

Address for correspondence:

L. Marinov, Dept. of Paediatrics and Medical genetics, Medical

University of Varna, 55 Marin Drinov str., BG-9002, Varna

e-mail:marinovvs@abv.bg

\section{MATERIALS AND METHODS}

For eight years (2000 - 2008 year) were hospitalized 134 children with high levels of blood pressure, from the age of 4 to 18 years.

All the children had familial history of cardio-vascular, brain-vascular diseases, arterial hypertension and other diseases, which can cause hypertonic conditions. A clinical examination was performed and the blood pressure was measured according to the accepted methodology (5).

All the children underwent laboratory, functional, image and other tests for the etiological specifying of the arterial hypertension.

\section{RESULTS AND DISCUSSION}

From the hospitalized 134 children with high blood pressure, in $20(14,18 \%), 11$ girls and 9 boys, we found that this is a symptomatic arterial hypertension. The smallest child was 4 years old, and the eldest 17 years old. The distribution of the cases according to the etiological belonging and sex is given in table 1 .

Familial history of arterial hypertension, ischemic heart disease and brain-vascular disease of first and second generation had 14 of the children. Additional familial history of 
diseases of the urinary system had 8 children, as 2 of them had no history of arterial hypertension.

\begin{tabular}{||l|c|c|c|c||}
\hline \hline SAH & Renovasal & $\begin{array}{c}\text { Suprarenal } \\
\text { tumor }\end{array}$ & $\begin{array}{c}\text { Renal } \\
\text { polycystosis }\end{array}$ & $\begin{array}{c}\text { Renal } \\
\text { parenchym } \\
\text { al disease }\end{array}$ \\
\hline Girls & 0 & 1 & 2 & 8 \\
\hline Boys & 2 & 0 & 1 & 6 \\
\hline \hline
\end{tabular}

For the presence of SAH we were directed because of the poor response of the blood pressure after the usual antihypertensive treatment. Three of the children were hospitalized in severe condition, after hypertonic crisis and one of them was with signs of hypertonic encephalopathy.

The frequency of SAH connected with renal disease is the highest, as is seen in the table. Two of the children with parenchymal renal disease were in advanced stages, with signs of renal insufficiency, as one of them was on haemodialysis. During the stay in the clinic it had sudden rises of the blood pressure, with signs of hypertonic encephalopathy, which led to the inclusion of fast acting vasodilatation drug (Naniprus).

In two of the children with reno-vasal hypertension there was stenosis of the renal arteria, as in one of them it was more severe, which necessitated the transfer of the child in the surgery for operative treatment. In one of the cases with SAH we found suprarenal tumor. The child was accepted in severe condition with hypertonic crisis, which was the first clinical sign of the disease. The levels of the blood pressure were influenced inadequately, besides the triple drug combination of antihypertensive drugs and diuretics. In this child, after the abdominal ultrasound, was suspected the presence of tumor formation, which was confirmed by computer tomography. The child was sent for surgical treatment. The histology of the extirpated tumor mass confirmed that this was a phaeochromocytoma.

In the other children with renal polycystosis and renal parenchimal disease, the levels of the blood pressure were raised moderately and were well influenced by the antihypertensive treatment. With the improvement of the main disease, the blood pressure showed tendency of normalization, which allowed the conduction of monotherapy with comparatively low doses.

In the literature is described that the most dramatic is the flow of SAH of reno-vasal origin and pheochromocytoma. Exactly these kinds of symptomatic hypertension have the most dramatic flow and are resistant to the treatment (6). The characteristic hypertonic crises, because of marked stenosis of the trunk of the renal arteria, were seen in our patient (2).

From the cases with parenchymal renal diseases, only in one child, the disease was with more dramatic flow - with signs of hypertonic encephalopathy. The child had chronic nephritis and advanced stages of renal insufficiency. Unfortunately the child died because of unseizured chronic renal insufficiency.

In conclusion, we may say, that the cases of SAH during childhood, which we have observed, are in the ranges of the average cited in the literature. In difference of the children with essential arterial hypertension, these with symptomatic hypertension, demonstrate more severe clinical manifestation of the disease. In some cases the clinical manifestation of high arterial blood pressure can be masked of the main disease. Sometimes if we don't take a careful history, don't make a detailed clinical examination, the severity and main reason for the condition can be underestimated. A hypertonic encephalopathy, with disorders of consciousness and convulsions, may be assessed as an acute neurological condition, epileptic convulsion, etc., if we don't measure the arterial blood pressure of the child. Unfortunately in the examination of a child sometimes is omitted the measurement of blood pressure, which in some cases can give fast orientation to the right diagnostic investigation.

The Symptomatic Arterial Hypertension is a serious disease, in some cases leading to life threatening complications. It is obligatory to make etiological specification of $\mathrm{SAH}$. On this depends the future behavior. After establishing of the main disease, leading to the occurrence of hypertension, if it is curable, there can be practically full recovery of the hypertension.

\section{BIBLIOGRAPHY}

1. Bartosh S., Aronson A. Childhood hypertension. An update on etiology, diagnosis, and treatment. Pediatr Clin North Am. 1999; 46: 235-52.

2. Flynn J. Differentiation Between Primary and Secondary Hypertension in Children Using Ambulatory Blood Pressure Monitoring. Pediatrics 2002; 110;.(1): 89-93.

3. Flynn J. Evaluation and management of hypertension in childhood. Prog Pediatr Cardiol. 2001;12: 177-88.

4. Flynn J. Hypertension in adolescents. Adolesc Med Clin. 2005;16:11-29.)

5. National High Blood Pressure Education Program Working Group on High Blood Pressure in Children and Adolescents. The fourth report on the diagnosis, evaluation, and treatment of high blood pressure in children and adolescents. Pediatrics. 2004; 114 (2 suppl 4th Report): 555-576

6. Silverstein DM, Champoux E, Aviles DH, Vehaskari VM. Treatment of primary and secondary hypertension in children. Pediatr Nephrol. 2006; 21: $820-27$. 